\title{
DYNAMIC MODEL OF MULTI-RIGID-BODY SYSTEMS BASED ON PARTICLE DYNAMICS WITH RECURSIVE APPROACH
}

\author{
HAZEM ALI ATTIA
}

Received 28 September 2004 and in revised form 17 August 2005

A dynamic model for multi-rigid-body systems which consists of interconnected rigid bodies based on particle dynamics and a recursive approach is presented. The method uses the concepts of linear and angular momentums to generate the rigid body equations of motion in terms of the Cartesian coordinates of a dynamically equivalent constrained system of particles, without introducing any rotational coordinates and the corresponding rotational transformation matrix. For the open-chain system, the equations of motion are generated recursively along the serial chains. A closed-chain system is transformed to open-chain by cutting suitable kinematical joints and introducing cut-joint constraints. An example is chosen to demonstrate the generality and simplicity of the developed formulation.

\section{Introduction}

There are different formulations for the dynamic analysis of spatial mechanisms which vary in the system of coordinates used and in the way they introduce kinematical constraint equations $[8,9,14,17,18]$. Each formulation has its own advantages and disadvantages depending on the application. Some formulations are developed using a twostep transformation which leads to a simple and reduced system of equations. One method $[13,16]$ uses initially the absolute coordinate formulation where the location of each rigid body in the system is described in terms of a set of translational and rotational coordinates. Then, the equations of motion are transformed to a reduced set in terms of the relative joint variables. Another method uses initially the point coordinate formulation in which a dynamically equivalent constrained system of particles replaces the rigid bodies $[1,2,3,4,5,6,7,10,15]$. The global motion of the constrained system of particles together with the constraints imposed upon them represent both the translational and rotational motions of the rigid body. The external forces and couples acting on the body are distributed over the system of particles. Then, the equations of motion which are expressed in terms of the Cartesian coordinates of the particles are rederived in terms of the relative joint variables. The main disadvantage of this two-step transformation is 
the necessity to transform at every time step from the joint variables to the original system which is time consuming.

The purpose of any multibody dynamic formulation is to write mathematical model to represent the dynamic response of the multibody system. Since the multibody system consists of large number of bodies, it is important to search for efficient formulation. Two main approaches to reach this formulation which are known as the matrix or recursive approaches $[8,18]$. According to the construction of the multibody system, every approach has some advantages over the other. It is known that in the case of long chain of bodies, the recursive formulation becomes more efficient than the matrix formulation $[8,18]$. However, for short chains with many closed loops, the matrix formulation is more efficient than the recursive one. Therefore, the recursive formulation is more mathematical in nature than the matrix formation.

A recursive dynamical formulation for the dynamic analysis of planar mechanisms is presented by Attia [7]. The concepts of linear and angular momentum are used to write the rigid body dynamical equations without the need to distribute the external forces and couples over the particles. The method can be applied to recursively generate the equations of motion for open and/or closed-chain systems.

In this paper, a dynamic model for multi-rigid-body systems which consists of interconnected of rigid bodies is presented based on particle dynamics and a recursive approach. The method is based upon the idea of replacing the rigid body by its dynamically equivalent constrained system of particles discussed in $[1,2,4,10,15]$ with essential modifications and improvements. In the previous work $[1,2,4,10,15]$, a matrix formulation was developed, where in the present formulation, a recursive formulation is deduced. Therefore, the two approaches and therefore, the resulting form of the equations are essentially different. In the present work, the concepts of the linear and angular momentums are used to formulate the rigid body dynamical equations. However, they are expressed in terms of the rectangular Cartesian coordinates of the equivalent constrained system of particles. This groups the advantages of the automatic elimination of the unknown internal forces as in Newton-Euler formulation and results in a reduced system of differential-algebraic equations. Some useful geometrical relationships are used to obtain a reduced dynamically equivalent constrained system of particles.

For the open-chain system, the equations of motion are generated recursively along the serial chains instead of the matrix formulation derived in $[1,2,3,4,5,6,7,10,15]$. Most of the kinematical constraints due to the kinematical joints are automatically eliminated by properly locating the equivalent particles. For the closed-chain system, the system is transformed to open-chain system by cutting suitable kinematical joints and introducing the cut-joint kinematical constraints. Examples are chosen to demonstrate the generality and simplicity of the proposed method.

\section{The dynamic formulation}

2.1. Construction of the equivalent system of particles. A rigid body and its dynamically equivalent constrained system of particles should have the same mass, the position of the centre of mass and the inertia tensor with respect to a body attached coordinate frame which results in ten conditions in the spatial case. The choice of four particles (not 


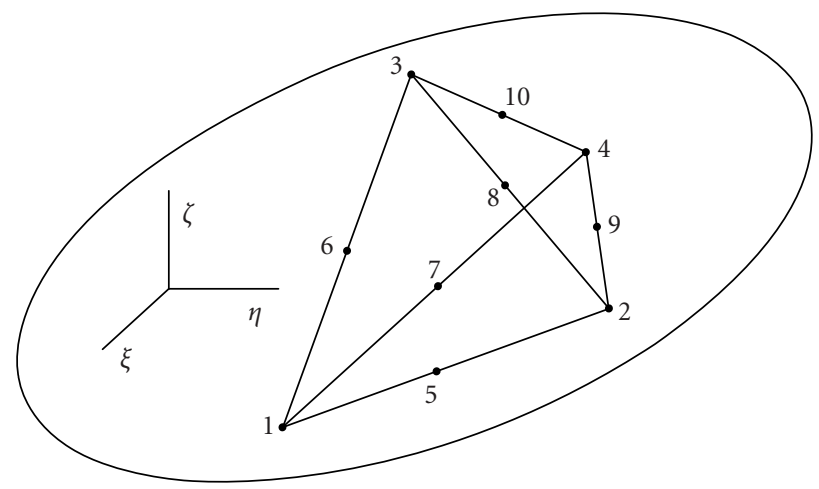

Figure 2.1. The rigid body system with the equivalent system of ten particles.

all are laying in the same plane) results in 16 unknowns ( 4 masses +12 coordinates) that should satisfy the ten conditions. However, this choice will lead to the solution of nonlinear algebraic equations due to the quadratic form of the second moments and also it does not give the freedom to choose all the particles in important places in the mechanisms. A system of ten particles will lead to the solution of ten linear algebraic equations in ten unknown masses. Also it gives the freedom of positioning the particles on the bodies in accordance with the joints that connect the bodies in order to reduce the number of particles and consequently eliminate some geometric and kinematical constraints. Therefore, a system of ten particles is chosen to replace the rigid body as shown in Figure 2.1. It constitutes four particles $1, \ldots, 4$, which are denoted as primary particles and six additional particles $5, \ldots, 10$, which are denoted as secondary particles. Each secondary particle is located at the middle point between a pair of primary particles in order to facilitate the elimination of its acceleration components and, in turn, to reduce the unknown motion variables. The mass distributions to points must satisfy the following conditions:

$$
\begin{gathered}
m=\sum_{i=1}^{10} m_{i}, \\
m \bar{r}_{G}=\sum_{i=1}^{10} m_{i} \bar{r}_{i}, \\
I_{\xi \xi}=\sum_{i=1}^{10} m_{i}\left(\zeta_{i}^{2}+\eta_{i}^{2}\right), \\
I_{\eta \eta}=\sum_{i=1}^{10} m_{i}\left(\zeta_{i}^{2}+\xi_{i}^{2}\right), \\
I_{\zeta \zeta}=\sum_{i=1}^{10} m_{i}\left(\xi_{i}^{2}+\eta_{i}^{2}\right),
\end{gathered}
$$




$$
\begin{gathered}
I_{\xi \eta}=\sum_{i=1}^{10} m_{i} \xi_{i} \eta_{i}, \\
I_{\xi \zeta}=\sum_{i=1}^{10} m_{i} \xi_{i} \zeta_{i}, \\
I_{\zeta \eta}=\sum_{i=1}^{10} m_{i} \zeta_{i} \eta_{i},
\end{gathered}
$$

where $m$ is the mass of the body, $\bar{r}_{G}$ is the position vector of the centre of mass of the body with respect to the body attached coordinate frame, $I_{\xi \xi}, I_{\eta \eta}, I_{\zeta \zeta}$ are the moments of inertia of the body with respect to the body attached coordinate frame, $I_{\xi \eta}, I_{\xi \zeta}, I_{\zeta \eta}$ are the products of inertia of the body with respect to the body attached coordinate frame, $m_{i}$ is the mass of particle $i$, and $\bar{r}_{i}$ is the position vector of particle $i$ with respect to the attached coordinate frame. Equation (2.1) represents a $10 \times 10$ linear system of algebraic equations in 10 unknown masses of the primary and secondary particles. At the same time, the coordinates of the particles can be chosen arbitrarily, which gives the advantage of the automatic elimination of the kinematical constraints due to some mechanical joints. Also it allows for two adjacent rigid bodies to contribute to the mass concentrated at the joint connecting them which reduces the total number of particles replacing the whole system.

2.2. Equations of motion of a single rigid body in spatial motion. Consider a rigid body which is acted upon by external forces and force couples. The rigid body is replaced by an equivalent system of ten particles. The distances between the ten particles are invariants as a result of the internal forces existing between them. The vector sum of these unknown internal forces or also the vector sum of their moments about any point equals zero by the law of action and reaction [12]. Then, the linear momentum equation for the whole system of particles yields,

$$
R=\sum_{i=1}^{10} m_{i} \ddot{r}_{i}
$$

where $\mathbf{R}$ is the vector sum of the external forces acting on the rigid body and $\ddot{r}_{i}$ is the acceleration vector of particle $i$. Also, the angular momentum equation for the whole system of particles with respect to particle 1 results in [12]

$$
G_{1}=\sum_{i=2}^{10} m_{i} r_{i, 1} \wedge \ddot{r}_{i}=\sum_{i=2}^{10} m_{i} \tilde{r}_{i, 1} \ddot{r}_{i}
$$

where $G_{1}$ is the vector sum of the moments of the external forces and force couples acting on the body with respect to particle 1 and $r_{i, 1}$ is the relative position vector between particles $i$ and 1 and the tilde sign above $r_{i, 1}$ is a consequence of an outer product with the vector which follows. The distance constraints between the ten particles are given as 
(see Figure 2.1)

$$
\begin{aligned}
& r_{2,1}^{T} r_{2,1}-d_{2,1}^{2}=0, \\
& r_{4,1}^{T} r_{4,1}-d_{4,1}^{2}=0, \\
& r_{4,2}^{T} r_{4,2}-d_{4,2}^{2}=0, \\
& r_{3,1}^{T} r_{3,1}-d_{3,1}^{2}=0, \\
& r_{3,2}^{T} r_{3,2}-d_{3,2}^{2}=0, \\
& r_{3,4}^{T} r_{3,4}-d_{3,4}^{2}=0, \\
& r_{5}-\left(r_{1}+r_{2}\right) / 2=0, \\
& r_{6}-\left(r_{1}+r_{3}\right) / 2=0, \\
& r_{7}-\left(r_{1}+r_{4}\right) / 2=0, \\
& r_{8}-\left(r_{2}+r_{3}\right) / 2=0, \\
& r_{9}-\left(r_{2}+r_{4}\right) / 2=0, \\
& r_{10}-\left(r_{3}+r_{4}\right) / 2=0,
\end{aligned}
$$

where the masses $m_{5}, m_{6}, m_{7}, m_{8}, m_{9}, m_{10}$ are located, respectively, at the middle point of masses $m_{1}$ and $m_{2}, m_{1}$ and $m_{3}, m_{1}$ and $m_{4}, m_{2}$ and $m_{3}, m_{2}$ and $m_{4}$, and $m_{3}$ and $m_{4}$ and $d_{i, j}$ is the distance between particles $i$ and $j$. The equations of motion (2.2), (2.3) and the constraint equations (2.4) represent a system of differential-algebraic equations that can be solved to determine the unknown acceleration vectors $\ddot{r}_{i}$ of the particles at any instant of time. However, due to the large number of the geometric constraints the integration of these equations is inefficient. In the following section, some useful geometrical relationships are used to eliminate the majority of these constraints.

2.3. The reduced form of the equations of motion of a single rigid body. The reduced form of the equations of motion can be achieved in two steps. First, the accelerations of the secondary particles and their unknown accelerations can be easily eliminated by substituting the constraint equations (2.4g) to (2.4l) into (2.2) and (2.3) to obtain

$$
R=\sum_{i=1}^{4} \bar{m}_{i} \ddot{r}_{i}, \quad G_{1}=\sum_{i=1}^{4} A_{i} \ddot{r}_{i},
$$

where

$$
\begin{gathered}
\bar{m}_{i}=m_{i}+\sum_{j=1, j \neq i}^{4} \frac{1}{2} m_{i, j}, \\
A_{i}=\overline{\bar{m}}_{i} \tilde{r}_{i, 1}+\sum_{j=2, j \neq i}^{4} \frac{1}{4} m_{i, j} \tilde{r}_{j, 1}, \\
\overline{\bar{m}}_{i}=m_{i}+\sum_{j=1, j \neq i}^{4} \frac{1}{4} m_{i, j},
\end{gathered}
$$


and where $m_{i, j}$ denotes the mass of the secondary particle that is located between the primary particles $i$ and $j\left(m_{1,2}=m_{5}, \ldots\right.$ etc.). Then, (2.5) in addition to the remaining constraints equations (2.4a) to (2.4f) represent the equations of motion for a single rigid body where only the accelerations of the primary particles stay.

A more reduced set of equations of motion can be derived by expressing the position vector of one of the primary particles in terms of the position vectors of the other three primary particles. We choose to express the coordinates of particle 3 in terms of the coordinates of particles 1,2, and 4. As shown in Figure 2.2, three invariant quantities $\bar{\lambda}$, $\bar{\mu}$, and $\bar{\tau}$ can be estimated with the aid of the constraint equations (2.4d) to (2.4f) that fix the distances between particle 3 and particles 1,2, and 4, respectively. The invariant quantities take the form,

$$
\begin{gathered}
\bar{\lambda}=\mid \frac{r_{3,1}^{T} \tilde{r}_{2,1} r_{4,1}}{\left|\tilde{r}_{2,1} r_{4,1}\right|}, \\
\bar{\nu}=\sqrt{\left|r_{3,1}\right|^{2}-\bar{\lambda}^{2}}, \\
\bar{\mu}=\frac{\left|r_{4,2}\right|\left|\tilde{r}_{2,1} r_{5,1}\right|}{\left|\tilde{r}_{5,1} r_{4,2}\right|}, \\
\bar{\tau}=\left|r_{4,2}\right|-\bar{\mu},
\end{gathered}
$$

where

$$
r_{\overline{5}, 1}=r_{3,1}-\bar{\lambda} \frac{\tilde{r}_{2,1} r_{4,1}}{\left|\tilde{r}_{2,1} r_{4,1}\right|} .
$$

Knowing the initial Cartesian coordinates of the primary particles, the invariant quantities are determined using (2.7). In terms of these invariant quantities, the position vector of particle 3 is expressed as

$$
r_{3}=r_{1}+\bar{\lambda} \frac{\tilde{r}_{2,1} r_{4,1}}{\left|\tilde{r}_{2,1} r_{4,1}\right|}+\bar{\nu} \frac{\bar{\mu} r_{4,1}+\bar{\tau} r_{2,1}}{\left|\bar{\mu} r_{4,1}+\bar{\tau} r_{2,1}\right|} .
$$

Since the quantities in the denominators in the right hand side of (2.9) are invariants we can rearrange the terms and obtain the simpler form

$$
r_{3}=r_{1}+\lambda \tilde{r}_{2,1} r_{4,1}+\mu r_{4,1}+\tau r_{2,1}
$$

where

$$
\lambda=\frac{\bar{\lambda}}{\left|\tilde{r}_{2,1} r_{4,1}\right|}, \quad \mu=\frac{\bar{\nu} \bar{\mu}}{\left|\bar{\mu} r_{4,1}+\bar{\tau} r_{2,1}\right|}, \quad \tau=\frac{\bar{\nu} \bar{\tau}}{\left|\bar{\mu} r_{4,1}+\bar{\tau} r_{2,1}\right|} .
$$

The corresponding velocity and acceleration vectors of particle 3 are estimated using the first and second time differentiations of (2.10) respectively which result in the 


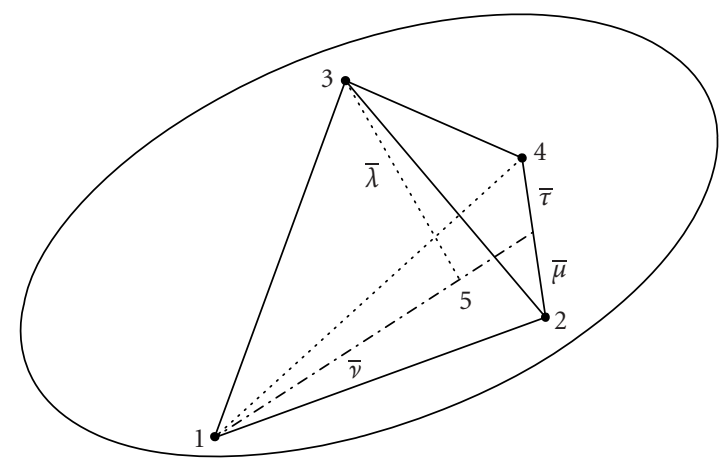

Figure 2.2. The rigid body system with its equivalent primary particles indicating the invariant quantities.

following forms,

$$
\begin{aligned}
& \dot{r}_{3}=\dot{r}_{1}+\lambda\left(\tilde{r}_{2,1} \dot{r}_{4,1}+\dot{\tilde{r}}_{2,1} r_{4,1}\right)+\mu \dot{r}_{4,1}+\tau \dot{r}_{2,1}, \\
& \ddot{r}_{3}=\ddot{r}_{1}+\lambda\left(\tilde{r}_{2,1} \ddot{r}_{4,1}+\ddot{\tilde{r}}_{2,1} r_{4,1}+2 \dot{\tilde{r}}_{2,1} \dot{r}_{4,1}\right)+\mu \ddot{r}_{4,1}+\tau \ddot{r}_{2,1} .
\end{aligned}
$$

Equation (2.13) expresses the unknown acceleration vector of particle 3 in terms of the acceleration vectors of the other primary particles which eliminates the constraint equations (2.4d) to (2.4f). Equation (2.13) can be put in the more convenient form,

$$
\ddot{r}_{3}=\left(1-\mu-\tau+\lambda \tilde{r}_{4,2}\right) \ddot{r}_{1}+\left(\tau-\lambda \tilde{r}_{4,1}\right) \ddot{r}_{2}+\left(\mu+\lambda \tilde{r}_{2,1}\right) \ddot{r}_{4} .
$$

Substituting the derived acceleration vector of particle 3 from (2.14) into (2.5), then the differential equations of motion take the modified form

$$
\begin{aligned}
R=\{ & \left.\bar{m}_{1}+\bar{m}_{3}\left(1-\mu-\tau+\lambda \tilde{r}_{4,2}\right)\right\} \ddot{r}_{1} \\
& +\left\{\bar{m}_{2}+\bar{m}_{3}\left(\tau-\lambda \tilde{r}_{4,1}\right)\right\} \ddot{r}_{2}+\left\{\bar{m}_{4}+\bar{m}_{3}\left(\mu+\lambda \tilde{r}_{2,1}\right)\right\} \ddot{r}_{4}+2 \lambda \bar{m}_{3} \dot{\tilde{r}}_{2,1} \dot{r}_{4,1}, \\
G_{1}= & \left\{A_{1}+A_{3}\left(1-\mu-\tau+\lambda \tilde{r}_{4,2}\right)\right\} \ddot{r}_{1} \\
& +\left\{A_{2}+A_{3}\left(\tau-\lambda \tilde{r}_{4,1}\right)\right\} \ddot{r}_{2}+\left\{A_{4}+A_{3}\left(\mu+\lambda \tilde{r}_{2,1}\right)\right\} \ddot{r}_{4}+2 \lambda A_{3} \dot{\tilde{r}}_{2,1} \dot{r}_{4,1} .
\end{aligned}
$$

Equations (2.15) and (2.16) in addition to the constraint equations (2.4a) to (2.4c) represent the equations of motion of a single floating rigid body in spatial motion. It can be solved at every time step to determine the unknown acceleration components of particles 1,2, and 4. Consequently, (2.14) can be used to determine the acceleration components of particle 3 . The acceleration components of the particles are integrated numerically knowing their Cartesian coordinates and velocities at a certain time to determine the positions and velocities for the next time step. Gear's method [11] for the numerical integration of differential-algebraic equations is used to overcome the instability problem resulting during the modeling process of constraint mechanical systems. The motion of the particles determines completely the translational and rotational motion of the rigid body. If the rigid body is rotating about a fixed point, then particle 1 may be located at 


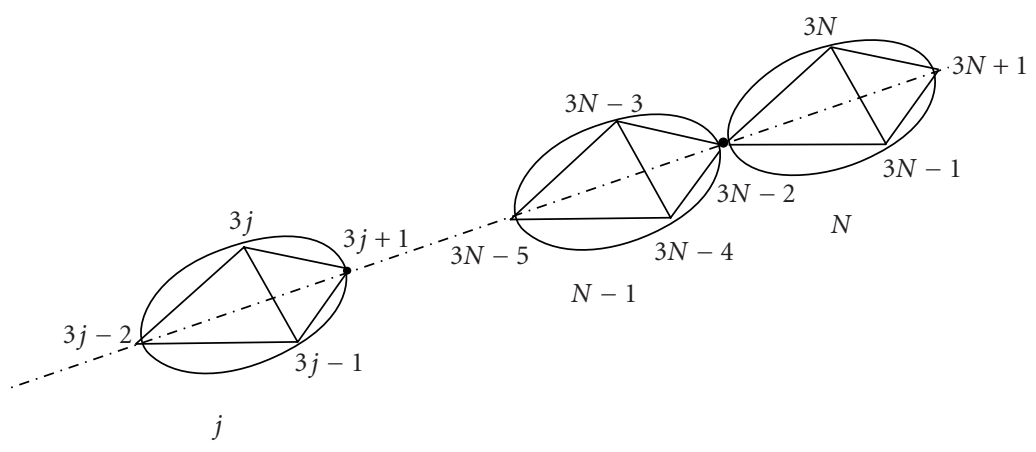

Figure 2.3. Serial chain of $N$ rigid bodies with the equivalent system of primary particles.

the centre of this joint. In this case, (2.16) and (2.4a) to (2.4c) are used to solve for the unknown Cartesian accelerations of particles 2 and 4. Equation (2.15) can be solved to determine the unknown reaction forces at the joint $N_{1}$ as,

$$
\begin{aligned}
N_{1}= & \left\{\bar{m}_{1}+\bar{m}_{3}\left(1-\mu-\tau+\lambda \tilde{r}_{4,2}\right)\right\} \ddot{r}_{1} \\
& +\left\{\bar{m}_{2}+\bar{m}_{3}\left(\tau-\lambda \tilde{r}_{4,1}\right)\right\} \ddot{r}_{2}+\left\{\bar{m}_{4}+\bar{m}_{3}\left(\mu+\lambda \tilde{r}_{2,1}\right)\right\} \ddot{r}_{4}+2 \lambda \bar{m}_{3} \dot{\tilde{r}}_{2,1} \dot{r}_{4,1}-R .
\end{aligned}
$$

If the rigid body is rotating about a fixed axis, then particles 1 and 2 can be located along the axis of the joint to define its direction. Then, to solve for the unknown acceleration vector of particle 4 , the constraint equations $(2.4 \mathrm{~b})$ and $(2.4 \mathrm{c})$ can be used in addition to one scalar moment equation that is generated by taking the projection of the vectors in (2.16) along the direction of the fixed axis. Then, (2.15) may be used to get the reactions at the axis of the revolute joint.

\subsection{Equations of motion of a serial chain of rigid bodies}

2.4.1. Case of a serial chain contains only spherical joints. Figure 2.3 shows a serial chain of $N$ rigid bodies connected by spherical joints with the equivalent system of $(3 N+1)$ particles where connected particles are unified from both bodies.

Since the recursive formulation developed here is suitable for computer implementation, it is then useful to write the equations of motion in the following organized steps.

Step 1. For the last body " $N$ " in the chain, the equations of motion are derived in a similar way as (2.16) and (2.4a) to (2.4c) of a single rigid body. The angular momentum equation takes the form

$$
\begin{aligned}
G_{N, 3 N-2}= & \left\{A_{3 N-2}+A_{3 N}\left(1-\mu_{N}-\tau_{N}+\lambda_{N} \tilde{r}_{3 N+1,3 N-1}\right)\right\} \ddot{r}_{3 N-2} \\
& +\left\{A_{3 N-1}+A_{3 N}\left(\tau_{N}-\lambda_{N} \tilde{r}_{3 N+1,3 N-2}\right)\right\} \ddot{r}_{3 N-1} \\
& +\left\{A_{3 N+1}+A_{3 N}\left(\mu_{N}+\lambda_{N} \tilde{r}_{3 N-1,3 N-2}\right)\right\} \ddot{r}_{3 N+1} \\
& +2 \lambda_{N} A_{3 N} \dot{\tilde{r}}_{3 N-1,3 N-2} \dot{r}_{3 N+1,3 N-2},
\end{aligned}
$$


where

$$
\begin{gathered}
A_{3 N}=\overline{\bar{m}}_{3 N} \tilde{r}_{3 N, 3 N-2}+\sum_{i=3 N-1, i \neq 3 N}^{3 N+1} \frac{1}{4} m_{3 N, i} \tilde{r}_{i, 3 N-2}, \\
\overline{\bar{m}}_{3 N}=m_{3 N}+\sum_{i=3 N-2, i \neq 3 N}^{3 N+1} \frac{1}{4} m_{3 N, i},
\end{gathered}
$$

where $G_{N, 3 N-2}$ is the sum of the moments of the external forces and force couples acting on body $N$ with respect to the location of particle $3 N-2$. The acceleration equations of the distance constraint between primary particles belonging to body $N$ are given as

$$
\begin{aligned}
& r_{3 N-2,3 N-1}^{T} \ddot{r}_{3 N-2}+r_{3 N-1,3 N-2}^{T} \ddot{r}_{3 N-1}=-\dot{r}_{3 N-1,3 N-2}^{T} \dot{r}_{3 N-1,3 N-2}, \\
& r_{3 N-2,3 N+1}^{T} \ddot{r}_{3 N-2}+r_{3 N+1,3 N-2}^{T} \ddot{r}_{3 N+1}=-\dot{r}_{3 N+1,3 N-2}^{T} \dot{r}_{3 N+1,3 N-2}, \\
& r_{3 N-1,3 N+1}^{T} \ddot{r}_{3 N-1}+r_{3 N+1,3 N-1}^{T} \ddot{r}_{3 N+1}=-\dot{r}_{3 N+1,3 N-1}^{T} \dot{r}_{3 N+1,3 N-1} .
\end{aligned}
$$

Step 2. Addition of one more body in the chain leads to the inclusion of an angular momentum vector equation that takes into consideration the contributions of all the ascending bodies in the chain together with three distance constraint equations between the particles belonging to this body. These six scalar equations are appended to the equations of motion derived for the leading bodies in the chain. For body $j$, the appended equations of motion take the form

$$
\begin{aligned}
\sum_{k=j}^{N} G_{k, 3 j-2}= & \sum_{k=j}^{N}\left\{A_{3 k-2}+A_{3 k}\left(1-\mu_{k}-\tau_{k}+\lambda_{k} \tilde{r}_{3 k+1,3 k-1}\right)\right\} \ddot{r}_{3 k-2} \\
& +\left\{A_{3 k-1}+A_{3 k}\left(\tau_{k}-\lambda_{k} \tilde{r}_{3 k+1,3 k-2}\right)\right\} \ddot{r}_{3 k-1} \\
& +\left\{A_{3 k+1}+A_{3 k}\left(\mu_{k}+\lambda_{k} \tilde{r}_{3 k-1,3 k-2}\right)\right\} \ddot{r}_{3 k+1} \\
& +2 \lambda_{k} A_{3 k} \dot{\tilde{r}}_{3 k-1,3 k-2} \dot{r}_{3 k+!, 3 k-2},
\end{aligned}
$$

where

$$
\begin{gathered}
A_{3 k}=\overline{\bar{m}}_{3 k} \tilde{r}_{3 k, 3 k-1}+\sum_{i=3 k-1, i \neq 3 k}^{3 k+1} \frac{1}{4} m_{3 k, i} \tilde{r}_{i, 3 k-2}, \\
\overline{\bar{m}}_{3 k}=m_{3 k}+\sum_{i=3 k-2, i \neq 3 k}^{3 k+1} \frac{1}{4} m_{3 k, i}, \\
r_{3 j-2,3 j-1}^{T} \ddot{r}_{3 j-2}+r_{3 j-1,3 j-2}^{T} \ddot{r}_{3 j-1}=-\dot{r}_{3 j-1,3 j-2}^{T} \dot{r}_{3 j-1,3 j-2}, \\
r_{3 j-2,3 j+1}^{T} \ddot{r}_{3 j-2}+r_{3 j+1,3 j-2}^{T} \ddot{r}_{3 j+1}=-\dot{r}_{3 j+1,3 j-2}^{T} \dot{r}_{3 j+1,3 j-2}, \\
r_{3 j-1,3 j+1}^{T} \ddot{r}_{3 j-1}+r_{3 j+1,3 j-1}^{T} \ddot{r}_{3 j+1}=-\dot{r}_{3 j+1,3 j-1}^{T} \dot{r}_{3 j+1,3 j-1},
\end{gathered}
$$


where according to (2.14),

$$
\begin{aligned}
\ddot{r}_{3 k}= & \left(1-\mu_{k}-\tau_{k}+\lambda_{k} \tilde{r}_{3 k+1,3 k-1}\right) \ddot{r}_{3 k-2} \\
& +\left(\tau_{k}-\lambda_{k} \tilde{r}_{3 k+1,3 k-2}\right) \ddot{r}_{3 k-1}+\left(\mu_{k}+\lambda_{k} \tilde{r}_{3 k-1,3 k-2}\right) \ddot{r}_{3 k+1} .
\end{aligned}
$$

If body " $j$ " is the floating base body in the chain then, three linear momentum equations, similar to (2.15), are required to solve for the unknown acceleration components of particle 1. These linear momentum equations equate the sum of the external forces acting on all the bodies in the chain to the time rate of change of the vectors of linear momentum of all the equivalent particles that replace the chain which take the form

$$
\begin{aligned}
\sum_{k=j}^{N} R= & \sum_{k=j}^{N}\left\{\bar{m}_{3 k-2}+\bar{m}_{3 k}\left(1-\mu_{k}-\tau_{k}+\lambda_{k} \tilde{r}_{3 k+1,3 k-1}\right)\right\} \ddot{r}_{3 k-2} \\
& +\left\{\bar{m}_{3 k-1}+\bar{m}_{3 k}\left(\tau_{k}-\lambda_{k} \tilde{r}_{3 k+1,3 k-2}\right)\right\} \ddot{r}_{3 k-1} \\
& +\left\{\bar{m}_{3 k+1}+\bar{m}_{3 k}\left(\mu_{k}+\lambda_{k} \tilde{r}_{3 k-1,3 k-2}\right)\right\} \ddot{r}_{3 k+1} \\
& +2 \lambda_{k} \bar{m}_{3 k} \dot{\tilde{r}}_{3 k-1,3 k-2} \dot{r}_{3 k+1,3 k-2}
\end{aligned}
$$

where

$$
\bar{m}_{3 k}=m_{3 k}+\sum_{i=3 k-2, i \neq 3 k}^{3 k+1} \frac{1}{2} m_{3 k, i} .
$$

In general, for a serial chain of $N$ bodies, an equivalent system of $(3 N+1)$ primary particles and $6 \mathrm{~N}$ secondary particles is first constructed. Then, by eliminating all the accelerations of the secondary particles and $N$ primary particles, we are left with $2 N+1$ accelerations of the particles and consequently, $6 N+3$ unknown acceleration components. To solve for these unknowns, $3 \mathrm{~N}$ angular momentum equations can be generated recursively along the chain together with $3 \mathrm{~N}$ distance constraints between the particles located on each body. Finally, three linear momentum equations can be used to solve for the unknown acceleration components of particle 1 if body 1 is floating or for the unknown reaction forces if there is a fixation at point 1 .

Step 3. If body $j$ is connected to body $j-1$ by a revolute joint, then we take the projection of all the moment vectors in (2.21) along the axis of the joint which is defined by two particles from both bodies that are commonly located on it. Two additional distance constraints, that fix the distances between the remaining fourth particle and the other two particles along the axis of the joint, together with the angular momentum equation can be used to solve for the acceleration vector of the fourth particle on body $j$.

Step 4. If bodies " $j$ " and " $j-1$ " in a serial chain are connected by a prismatic joint, then particles $3 j-5,3 j-4,3 j-3$, and $3 j-2$ are located on body " $j-1$ " while particles $3 j-1,3 j, 3 j+1$, and $3 j+2$ are assigned to body " $j$." Particles $3 j-5$ and $3 j-2$ on body " $j-1$ " and particles $3 j-1$ and $3 j+2$ on body " $j$ " are arbitrarily located along the axis of the prismatic joint. To obtain the equations of motion for body " $j$," one force equation can be written by taking the projection of all the vectors in (2.21) along the axis of 
the prismatic joint together with the distance constraint equations (2.24)-(2.25). Moreover, five independent kinematical constraint equations associated with the prismatic joint are included and take the form,

$$
\begin{gathered}
\left(r_{3 j-5}-r_{3 j-2}\right) x\left(r_{3 j-1}-r_{3 j+2}\right)=0, \\
\left(r_{3 j-5}-r_{3 j-2}\right) x\left(r_{3 j-1}-r_{3 j-2}\right)=0, \\
\left(r_{3 j-4,3 j-5}-\frac{r_{3 j-4,3 j-5}^{T} r_{3 j-2,3 j-5}}{\left|r_{3 j-2,3 j-5}\right|}\right)^{T}\left(r_{3 j, 3 j-1}-\frac{r_{3 j, 3 j-1}^{T} r_{3 j+2,3 j-1}}{\left|r_{3 j+2,3 j-1}\right|}\right)=0 .
\end{gathered}
$$

Therefore, for a preceding body " $h$ " in the chain the moment equation is generated recursively along the serial chain as addressed above which take the form,

$$
\begin{aligned}
\sum_{k=h}^{N} G_{k, 3 h-2}= & \sum_{k=h}^{j-1}\left\{A_{3 k-2}+A_{3 k}\left(1-\mu_{k}-\tau_{k}+\lambda_{k} \tilde{r}_{3 k+1,3 k-1}\right)\right\} \ddot{r}_{3 k-2} \\
& +\left\{A_{3 k-1}+A_{3 k}\left(\tau_{k}-\lambda_{k} \tilde{r}_{3 k+1,3 k-2}\right)\right\} \ddot{r}_{3 k-1} \\
& +\left\{A_{3 k+1}+A_{3 k}\left(\mu_{k}+\lambda_{k} \tilde{r}_{3 k-1,3 k-2}\right)\right\} \ddot{r}_{3 k+1} \\
& +2 \lambda_{k} A_{3 k} \dot{\tilde{r}}_{3 k-1,3 k-2} \dot{r}_{3 k+!, 3 k-2} \\
& +\sum_{k=j}^{N}\left\{A_{3 k-1}+A_{3 k+1}\left(1-\mu_{k}-\tau_{k}+\lambda_{k} \tilde{r}_{3 k+2,3 k}\right)\right\} \ddot{r}_{3 k-1} \\
& +\left\{A_{3 k}+A_{3 k+1}\left(\tau_{k}-\lambda_{k} \tilde{r}_{3 k+2,3 k-1}\right)\right\} \ddot{r}_{3 k} \\
& +\left\{A_{3 k+2}+A_{3 k+1}\left(\mu_{k}+\lambda_{k} \tilde{r}_{3 k, 3 k-1}\right)\right\} \ddot{r}_{3 k+2} \\
& +2 \lambda_{k} A_{3 k+1} \dot{\tilde{r}}_{3 k, 3 k-1} \dot{r}_{3 k+2,3 k-1} .
\end{aligned}
$$

If body " $h$ " is the floating base body in the chain, then a force equation, similar to (2.27), is written to solve for the unknown acceleration of particle 1 in the form,

$$
\begin{aligned}
\sum_{k=h}^{N} R_{k}= & \sum_{k=h}^{j-1}\left\{\bar{m}_{3 k-2}+\bar{m}_{3 k}\left(1-\mu_{k}-\tau_{k}+\lambda_{k} \tilde{r}_{3 k+1,3 k-1}\right)\right\} \ddot{r}_{3 k-2} \\
& +\left\{\bar{m}_{3 k-1}+\bar{m}_{3 k}\left(\tau_{k}-\lambda_{k} \tilde{r}_{3 k+1,3 k-2}\right)\right\} \ddot{r}_{3 k-1} \\
& +\left\{\bar{m}_{3 K=1}+\bar{m}_{3 k}\left(\mu_{k}+\lambda_{k} \tilde{r}_{3 k-1,3 k-2}\right)\right\} \ddot{r}_{3 k+1} \\
& +2 \lambda_{k} \bar{m}_{3 k} \dot{\tilde{r}}_{3 k-1,3 k-2} \dot{r}_{3 k+1,3 k-2} \\
& +\sum_{k=j}^{N}\left\{\bar{m}_{3 k-1}+\bar{m}_{3 k+1}\left(1-\mu_{k}-\tau_{k}+\lambda_{k} \tilde{r}_{3 k+2,3 k}\right)\right\} \ddot{r}_{3 k-1} \\
& +\left\{\bar{m}_{3 k}+\bar{m}_{3 k+1}\left(\tau_{k}-\lambda_{k} \tilde{r}_{3 k+2,3 k-1}\right)\right\} \ddot{r}_{3 k} \\
& +\left\{\bar{m}_{3 k+2}+\bar{m}_{3 k+1}\left(\mu_{k}+\lambda_{k} \tilde{r}_{3 k, 3 k-1}\right)\right\} \ddot{r}_{3 k+2} \\
& +2 \lambda_{k} \bar{m}_{3 k+1} \dot{\tilde{r}}_{3 k, 3 k-1} \dot{r}_{3 k+2,3 k-1} .
\end{aligned}
$$

Similar treatment can be used in dealing with all other kinds of lower or higher-pair kinematical joints [14]. 
2.4.2. Case of an open-chain or closed-chain. In the case of an open-chain system or closed-chain system, it can be transformed to a system of serial chains by cutting suitable joints and consequently cut-joint constraints are introduced. In the case of a closedchain system, the cut-joints avoid the need to introduce loop closure equations and the corresponding loop closure constraint forces and then allows the use of the laws of momentum/moment of momentum with respect to a joint axis. Equivalent particles are conveniently chosen to locate at the positions of the connection joints and in terms of their Cartesian coordinates the cut-joint constraint equations are easily formulated. The cutjoints kinematical constraints substitute for the unknown cut-joints constraint reaction forces that appear explicitly in the linear and angular momentum equations generated recursively along the separated serial chains.

It should be noted that in this formulation, the kinematical constraints due to some common types of kinematical joints (e.g., revolute or spherical joints) can be automatically eliminated by properly locating the equivalent particles. The remaining kinematical constraints along with the geometric constraints are, in general, either linear or quadratic in the Cartesian coordinates of the particles. Therefore, the coefficients of their Jacobian matrix are constants or linear in the rectangular Cartesian coordinates. Whereas in the formulation based on the relative coordinates [8], the constraint equations are derived based on loop closure equations which have the disadvantage that they do not directly determine the positions of the links and points of interest which makes the establishment of the dynamic problem more difficult. Also, the resulting constraint equations are highly nonlinear and contain complex circular functions. The absence of these circular functions in the point coordinate formulation leads to faster convergence and better accuracy. Furthermore, preprocessing the mechanism by the topological graph theory is not necessary as it would be the case with loop constraints.

Also, in comparison with the absolute coordinates formulation, the manual work of the local axes attachment and local coordinates evaluation as well as the use of the rotational variables and the rotation matrices in the absolute coordinate formulation are not required in the point coordinate formulation. This leads to fully computerized analysis and accounts for a reduction in the computational time and memory storage. In addition to that, the constraint equations take much simpler forms as compared with the absolute coordinates.

The elimination of the rotational coordinates, angular velocities and angular accelerations in the presented formulation, leads to possible savings in computation time when this procedure is compared against the absolute or relative coordinate formulation. It has been determined that numerical computations associated with rotational transformation matrices and their corresponding coordinate transformations between reference frames is time consuming and, therefore, if these computations are avoided more efficient codes may be developed [15]. The elimination of rotational coordinates can also be found very beneficial in design sensitivity analysis of multibody systems. In most procedures for design sensitivity analysis, leading to an optimal design process, the derivatives of certain functions with respect to a set of design parameters are required. Analytical evaluation of these derivatives are much simpler if the rotational coordinates are not present and if we only deal with translational coordinates. 
Some practical applications of multibody dynamics require one or more bodies in the system to be described as deformable in order to obtain a more realistic dynamic response [15]. Deformable bodies are normally modeled by the finite element technique. Assume that the deformable body is connected to a rigid body described by a set of particles. Then, one or more particles of the rigid body can coincide with one or more nodes of the deformable body in order to describe the kinematical joint between the two bodies. This is a much simpler process that when the rigid body is described by a set of translational and rotational coordinates. In general, the point coordinates have additional advantages over the other systems of coordinates since they are the most suitable coordinates for the graphics routines and the animation programs.

Also, since we are dealing in this formulation with a system of particles instead of rigid bodies, therefore only the laws of particle dynamics are utilized in generating the equations of motion of the mechanical system. This makes the formulation much simpler than the other dynamic formulations which use the rigid body dynamical equations of motion both translational and rotational. In summary, the methodologies presented in this paper have many interesting characteristics which may be found useful in some applications. These methodologies can be combined with other methods to develop even more efficient, accurate, and flexible procedures. It should be noted that there is no single multibody formulation to be considered as the best formulation for general multibody dynamics. Each formulation has its own unique or common features and, therefore, selected features should be adopted to our advantages [15].

2.5. Dynamic simulation of a vehicle with a wishbone double A-arms suspension. The model consists of a main chassis, two double A-arms suspension sub-systems, two simple A-arm suspension sub-systems, and four wheels as shown in Figure 2.4a. The front tires are connected to the main chassis by the simple A-arms suspensions. A suspension spring and a shock absorber are included in every suspension sub-system. The system has two kinematical closed loops in the front suspensions and two kinematical open loops in the rear suspensions. The whole vehicle has 16 degrees of freedom. The main chassis has six degrees of freedom, as a floating base body; three for the translational motion and three for the rotational motion. The front suspensions each has two degrees of freedom and the rear suspensions each has one degree of freedom. The four tires have four degrees of freedom corresponding to the rolling motion. Each tire is analytically modeled in the radial direction by an equivalent linear translational spring system which has damping characteristics. The inertia characteristics are presented in Table 2.1. The characteristics of the suspension springs and dampers, and the wheels are presented in Tables 2.2 and 2.3 , respectively.

This vehicle model is an example of an open/closed loop system. Each rigid body is represented by its equivalent system of particles. The main chassis is replaced by the fourprimary-particles representation. The knuckles, the front A-arms, and the rear A-arms each is replaced by the three-primary-particles representation. Each wheel is represented by the two-primary-particles representation along the wheel axis of every revolute joint to define its orientation and one particle is located at the centre of every spherical joint to define its location. The masses of these shared particles receive contributions from 


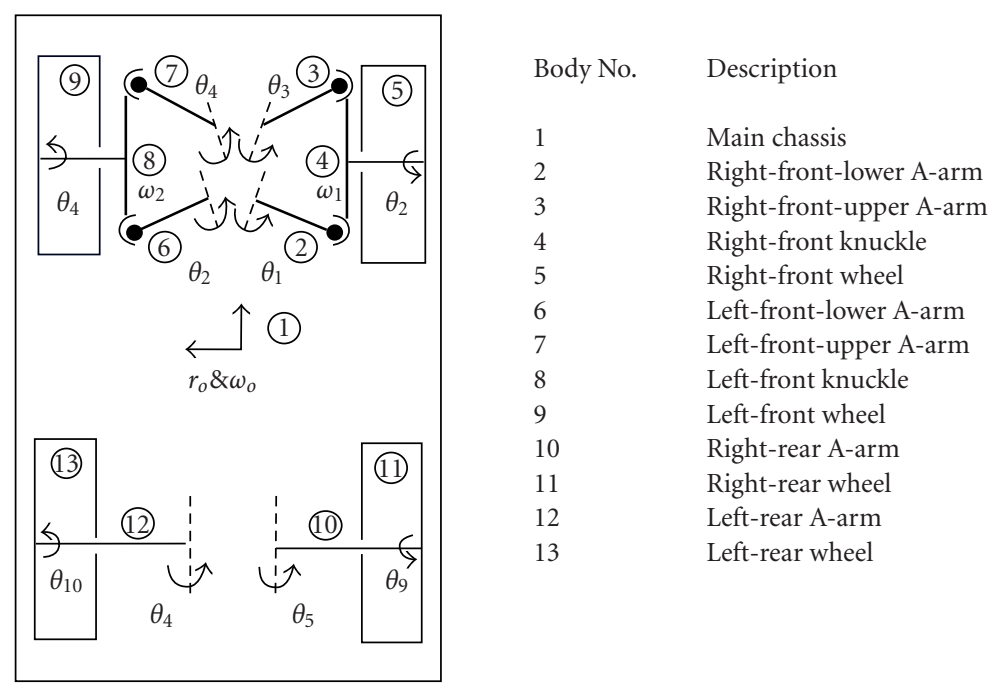

(a) Schematic view of the vehicle model with body numbers, joint types, and joint velocity variables.

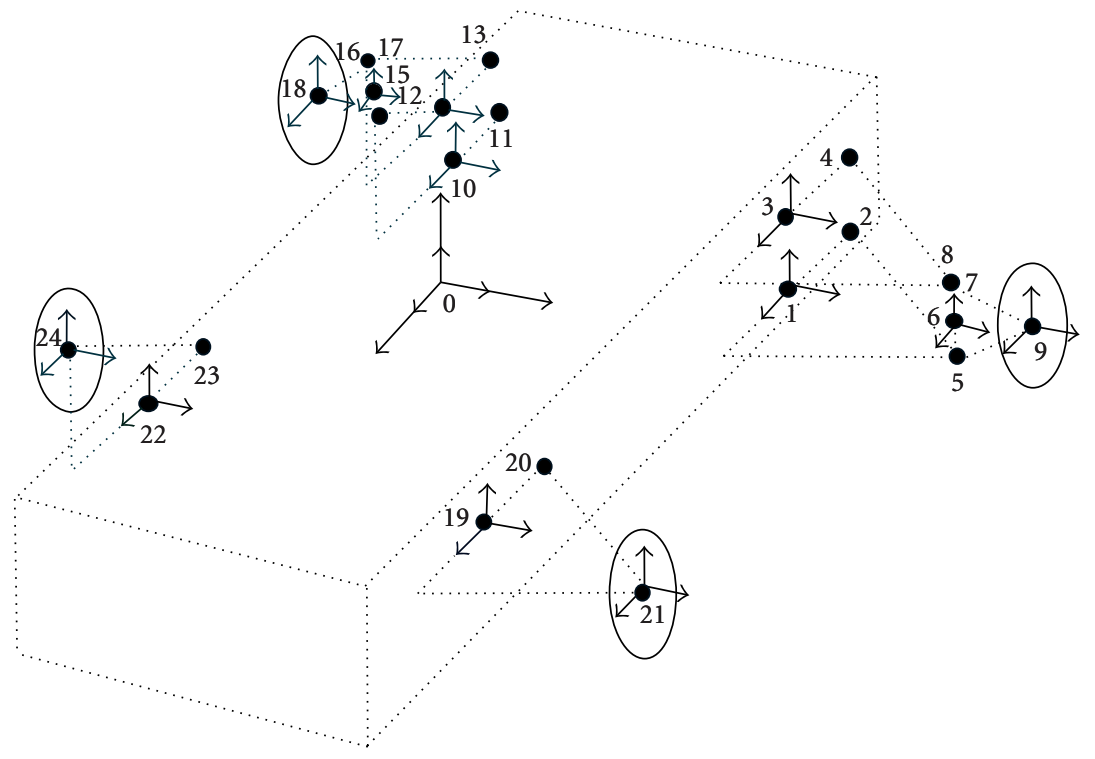

(b) Schematic diagram of the vehicle with the primary particles and the body attached coordinate frames.

Figure 2.4 
Table 2.1. Description of the rigid bodies.

\begin{tabular}{c|ccc}
\hline Body no. & Description & Mass $(\mathrm{kg})$ & $\begin{array}{c}\text { Inertia }\left(\mathrm{kg} \cdot \mathrm{m}^{2}\right) \\
\xi \xi, \eta \eta, \zeta \zeta, \eta \zeta, \xi \zeta, \xi \eta\end{array}$ \\
\hline 1 & Main chassis & 800.0 & $130,250,200,0,0,0$ \\
2 & Right-front-lower A-arm & 0.5 & $0.5,0.5,0.5,0,0,0$ \\
3 & Right-front-upper A-arm & 0.5 & $0.5,0.5,0.5,0,0,0$ \\
4 & Right-front knuckle & 5.0 & $1,1,1,0,0,0$ \\
5 & Right-front wheel & 20.0 & $2,3,2,0,0,0$ \\
10 & Right-rear A-arm & 0.5 & $0.5,0.5,0.5,0,0,0$ \\
11 & Right-rear wheel & 30.0 & $2,3,2,0,0,0$ \\
\hline
\end{tabular}

- Bodies in the left-hand side are symmetric to those in the right-hand side.

Table 2.2. Description of springs and dampers.

\begin{tabular}{c|cccc}
\hline No. & Connected bodies & $\mathrm{K}(\mathrm{N} / \mathrm{m})$ & $\mathrm{D}(\mathrm{N} . \mathrm{s} / \mathrm{m})$ & $1_{o}(\mathrm{~m})$ \\
\hline 1 & $(1,2)$ & $9.16 \mathrm{E}+04$ & $1.44 \mathrm{E}+04$ & 0.345 \\
2 & $(1,6)$ & $9.16 \mathrm{E}+04$ & $1.44 \mathrm{E}+04$ & 0.345 \\
3 & $(1,10)$ & $9.16 \mathrm{E}+04$ & $1.44 \mathrm{E}+04$ & 0.362 \\
4 & $(1,12)$ & $9.16 \mathrm{E}+04$ & $1.44 \mathrm{E}+04$ & 0.362 \\
\hline
\end{tabular}

Table 2.3. Description of tires.

\begin{tabular}{cc}
\hline Radius & $0.35 \mathrm{~m}$ \\
\hline Stiffness & $1.5 \mathrm{E}+05 \mathrm{~N} / \mathrm{m}$ \\
Damping coefficient & $1.3 \mathrm{E}+04 \mathrm{~N} . \mathrm{s} / \mathrm{m}$ \\
\hline
\end{tabular}

the two adjacent bodies. The whole vehicle is represented by a resultant system of 24 particles. For each rigid body, the locations of the particles are chosen arbitrary in reference to a centroidal local coordinate frame as shown in Figure 2.4b. The vehicle is divided into 3 independent serial branches: one branch is connecting an A-arm to the chassis to another A-arm and the other two branches each consists of a double A-arm suspension after cutting the joints connecting it to the chassis. Knowing the external forces applied to the vehicle and the initial coordinates and velocities of the different particles, then the equations of motion can be generated recursively along each branch as discussed in Section 2.4. Since the system consists of many rigid bodies in serial chains, it is advantages to use the recursive formulation developed here.

The motion of the vehicle is started from the rest position under the action of the gravitational forces and the wheels compression forces. Figures 2.5 and 2.6 show the time variations of the vertical displacement and acceleration of the center of the chassis respectively. Initially, the chassis is subjected to impulsive forces which result in a sudden change in the acceleration of its center. During time progression, due to the motion of the elements of the system, the variable force elements starts imposing constraint forces. Then, the chassis undergoes damped oscillations up to the steady state. 


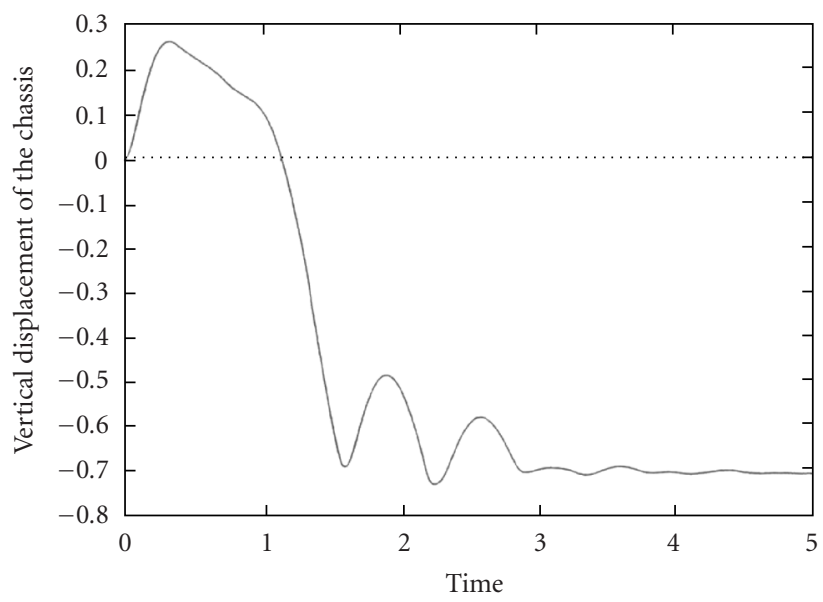

Figure 2.5. The time variation of the vertical displacement of the chassis (m).

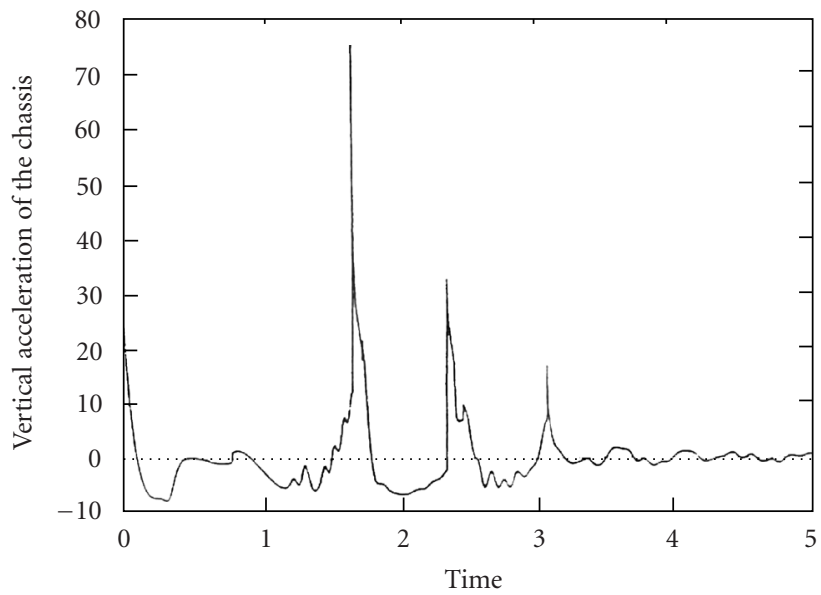

Figure 2.6. The time variation of the vertical acceleration of the chassis $\left(\mathrm{m} / \mathrm{s}^{2}\right)$.

\section{Conclusions}

In the present work, a recursive multi-rigid-body formulation for generating the equations of motion for the spatial motion of a system of rigid bodies is presented based on particle dynamics with recursive approach. The concepts of linear and angular momentums are used to formulate the rigid body dynamical equations of motion which are expressed in terms of the rectangular Cartesian coordinates of a dynamically equivalent constrained system of particles. This groups the advantages of the automatic elimination of the unknown internal constraint forces, the absence of any rotational coordinates in addition to the rotational transformation matrices, and the elimination of the necessity 
to distribute the external forces and force couples over the particles. Also, the formulation can be considered as a natural extension to the finite element representation for a deformable body. Some useful geometric relations are used which result in a reduced system of differential-algebraic equations. The formulation can be applied to open and/or closed-chain with the common types of kinematical joints. The developed formulation with its interesting characteristics may be found useful in some applications.

\section{References}

[1] H. A. Attia, A computer-oriented dynamical formulation with applications to multibody systems, Ph.D. dissertation, Department of Engineering Mathematics and Physics, Faculty of Engineering, Cairo University, Giza, 1993.

[2] - Formulation of the equations of motion for the RRRR robot manipulator, Transactions of the Canadian Society for Mechanical Engineers 22 (1998), no. 1, 83-93.

[3] _ A matrix formulation for the dynamic analysis of spatial mechanisms using point coordinates and velocity transformation, Acta Mech. 165 (2003), no. 3-4, 207-222.

[4] Numerical kinematic analysis of the standard MacPherson motor-vehicle uspension system, KSME International Journal, The Korean Society of Mechanical Engineers 17 (2003), no. 12, 1961-1968.

[5] - A computer method for the dynamic analysis of a system of rigid bodies in plane motion, KSME International Journal, The Korean Society of Mechanical Engineers 18 (2004), no. 2, 193-302.

[6] Dynamic analysis of a chain of rigid rods, KSIAM Journal, Korean Society for Industrial and Applied Mathematics 8 (2004), no. 2, 75-86.

[7] __ Equations of motion of planar mechanical systems based on particle dynamics and a recursive algorithm, Proceedings of the I MECH E. Part K: Journal of Multi-body Dynamics 218 (2004), no. 1, 31-38.

[8] J. Denavit and R. S. Hartenberg, A kinematic notation for lower-pair mechanisms based on matrices, Trans. ASME J. Appl. Mech. 22 (1955), 215-221.

[9] J. García de Jalón and E. Bayo, Kinematic and Dynamic Simulation of Multibody Systems, Mechanical Engineering Series, Springer, New York, 1994.

[10] J. García de Jalón, J. Unda, A. Avello, and J. M. Jimenez, Dynamic analysis of three-dimensional mechanisms in 'Natural' coordinates, ASME paper no. 86-DET-137, 1986.

[11] C. W. Gear, Differential-algebraic equation index transformations, SIAM J. Sci. Statist. Comput. 9 (1988), no. 1, 39-47.

[12] H. Goldstein, Classical Mechanics, Addison-Wesley, Massachusetts, 1950.

[13] S. S. Kim and M. J. Vanderploeg, A general and efficient method for dynamic analysis of mechanical systems using velocity transformation, ASME Journal of Mechanisms, Transmissions and Automation in Design 108 (1986), no. 2, 176-182.

[14] P. E. Nikravesh, Computer Aided Analysis of Mechanical Systems, Prentice-Hall, New Jersey, 1988.

[15] P. E. Nikravesh and H. A. Attia, Construction of the equations of motion for multibody dynamics using point and joint coordinates, Computer-Aided Analysis of Rigid and Flexible Mechanical Systems (Tróia, 1993), NATO Adv. Sci. Inst. Ser. E Appl. Sci., vol. 268, Kluwer Academic, Dordrecht, 1994, pp. 31-60.

[16] P. E. Nikravesh and G. Gim, Systematic construction of the equations of motion for multibody systems containing closed kinematic loops, Proc. ASME Design Automation Conference (Montreal, 1989), 1989, pp. 27-33. 


\section{Formulation of multi-rigid-body systems}

[17] N. Orlandea, M. A. Chace, and D. A. Calahan, A sparsity-oriented approach to dynamic analysis and design of mechanical systems, part I and II, ASME Journal of Engineering for Industry 99 (1977), 773-784.

[18] P. N. Sheth and J. J. Uicker Jr., IMP (Integrated Mechanisms Program), a computer aided design analysis system for mechanisms and linkage, ASME Journal of Engineering for Industry $\mathbf{9 4}$ (1972), no. 2, 454-464.

Hazem Ali Attia: Department of Engineering Mathematics and Physics, Faculty of Engineering, El-Fayoum University, El-Fayoum, Egypt

Current address: Department of Mathematics, College of Science, A-Qasseem University, P.O. Box 237, Buraidah 81999, KSA

E-mail address: ah1113@yahoo.com 


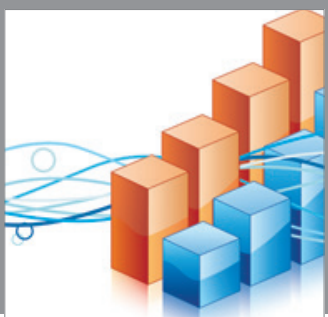

Advances in

Operations Research

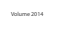

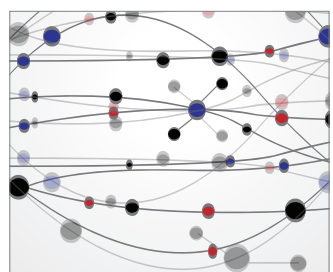

\section{The Scientific} World Journal
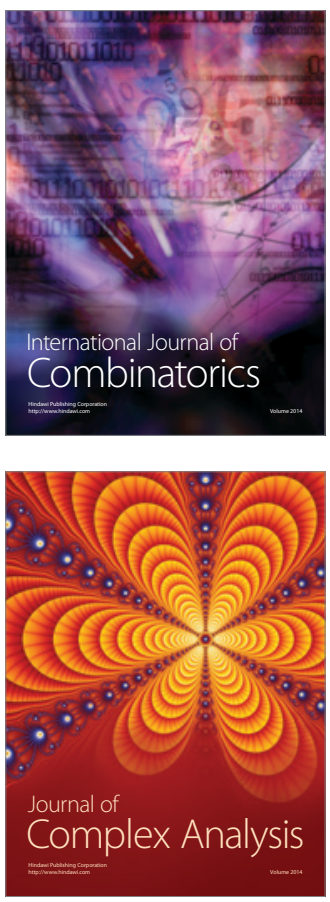

International Journal of

Mathematics and

Mathematical

Sciences
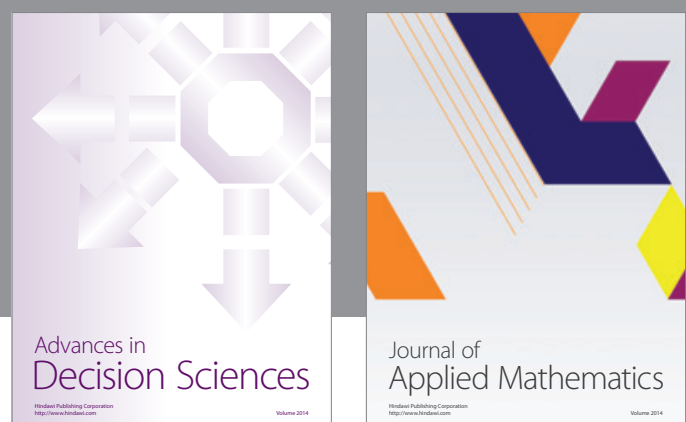

Journal of

Applied Mathematics
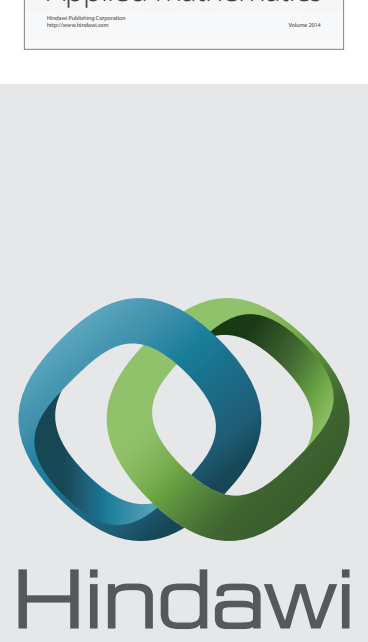

Submit your manuscripts at http://www.hindawi.com
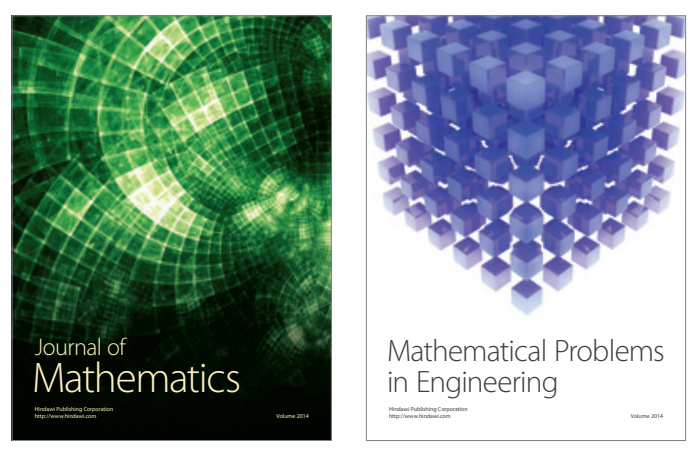

Mathematical Problems in Engineering
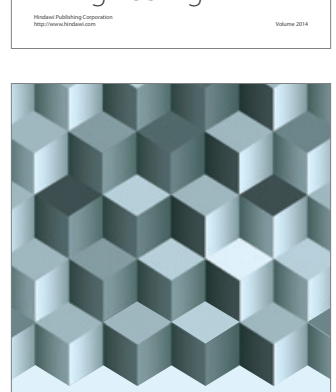

Journal of

Function Spaces
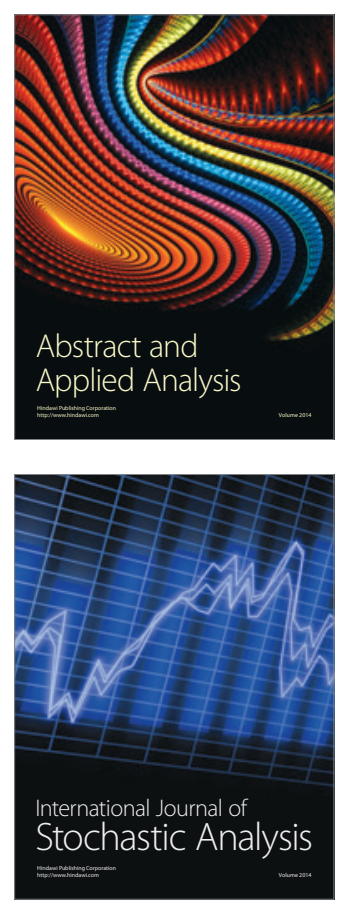

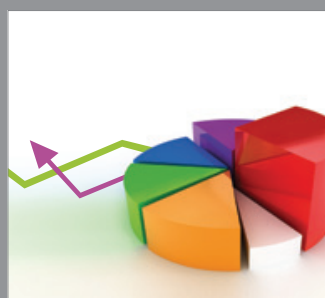

ournal of

Probability and Statistics

Promensencen
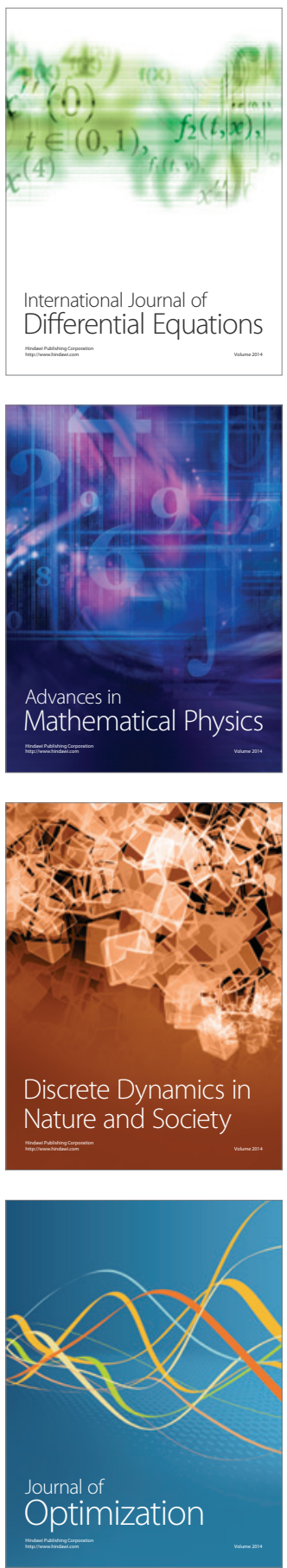